\title{
Cetyltrimethylammonium bromide inhibits the metastasis of breast cancer to the lungs by inhibiting epithelial-mesenchymal transition
}

\author{
Ning LI ${ }^{1, \#}$; YANG CHEN ${ }^{2, *}$; YongJie YANG ${ }^{3,4}$; SHUhAN LYU ${ }^{1}$; YUe PAN ${ }^{1,5, *}$ \\ ${ }^{1}$ State Key Laboratory of Fine Chemicals, Department of Pharmaceutical Sciences, School of Chemical Engineering, Dalian University of Technology, \\ Dalian, China \\ 2 Department of Pain Management, Shandong Provincial Hospital affiliated to Shandong First Medical University, Jinan, China \\ 3 Department of Pharmacy, The First Affiliated Hospital of Zhengzhou University, Zhengzhou, China \\ 4 Henan Key Laboratory of Precision Clinical Pharmacy, Zhengzhou University, Zhengzhou, China \\ ${ }^{5}$ Ningbo Institute of Dalian University of Technology, Ningbo, China
}

Key words: Migration, Effectiveness, Security, EMT-TFs

\begin{abstract}
Breast cancer is a highly aggressive cancer in females. Metastasis is a major obstacle to the efficient and successful treatment of breast cancer. Cetyltrimethylammonium bromide (CTAB) has anti-tumor effects on a variety of tumors. We showed that CTAB inhibits the metastasis of breast cancer to the lungs both in vitro and in vivo. Epithelial-mesenchymal transition (EMT) is thought to be one of the major processes mediating breast cancer metastasis. We found that CTAB suppressed EMT and regulated the levels of the classical EMT markers E-cadherin, $\mathrm{N}$-cadherin, vimentin, Snail and Twist1. Moreover, as a candidate anti-tumor agent, CTAB showed primary safety in vivo. Taken together, our results suggest that $\mathrm{CTAB}$ inhibits the migration of primary breast cancer to the lungs. Our findings confirm the clinical potential of CTAB for the treatment of breast cancer by targeting EMT. CTAB may thus be a promising novel anti-tumor drug for the treatment of breast cancer metastasis.
\end{abstract}

\section{Introduction}

Breast cancer is one of the most common neoplasms in women, and it has high potential for metastasis (Liang et al., 2020). Furthermore, the mortality rate is high in breast cancer patients due to metastasis and relapse (Rios Garcia et al., 2017). Although there are treatments available, such as surgery, chemotherapy and hormone therapy, breast cancer remains typified by a poor prognosis (Shah et al., 2018). Therefore, developing new therapies targeting metastasis is necessary to improve breast cancer outcomes.

The transition between epithelial cells and mesenchymallike cells occurs via a process known as epithelial-mesenchymal transition (EMT) (Gordan et al., 2007; Greenburg and Hay, 1982; Yang et al., 2019). It is believed that the EMT process confers malignancy to tumors and that the activation of EMT may initiate an invasion-metastasis cascade. In fact, EMT is closely related to the invasion and metastasis of a variety of cancers. During EMT, epithelial cells lose their adhesion

\footnotetext{
*Address correspondence to: Yue Pan, panyue0811@dlut.edu.cn

${ }^{\#}$ These authors have contributed equally to this work

Received: 13 July 2021; Accepted: 10 August 2021
}

junctions and acquire mesenchymal cell phenotypes (Koual et al., 2020), which are the main characteristics of aggressive and metastatic cancer cells. EMT can be stimulated by many factors, such as hypoxia and intracellular $\mathrm{pH}$ changes (Polyak and Weinberg, 2009). In response to these stimuli, several transcription factors, including E-cadherin, N-cadherin, Twist1, Snail and vimentin, are regulated and serve as important downstream mediators of EMT. A number of studies have reported that EMT-inducing transcription factors (EMT-TFs), such as Twist, Snail, Slug, and Zeb, are directly or indirectly involved in cancer cell metastasis (Fu et al., 2012; Tania et al., 2014). It is generally considered that Ecadherin inhibits EMT and acts as a suppressor of cancer invasion (Comijn et al., 2001; Lasagna et al., 2020). Zheng et al. (2018) reported that NRBE3 inhibits E-cadherin expression and further promotes breast cancer metastasis.

AMP-activated protein kinase (AMPK) is an evolutionarily conserved stress-sensing kinase that is activated under a variety of stresses, including hypoxia, ischemia, and nutrient deprivation. Furthermore, a change in the ATP:AMP ratio directly activates AMPK (Steinberg and Kemp, 2009). In addition to energy regulation, AMPK is also involved in a variety of physiological processes, including cell growth, cell division, autophagy, and cell migration. Several studies have 
linked AMPK to the migration and invasion of cancer cells (Chiu et al., 2009), and the activation of AMPK has been shown to prevent TGF- $\beta$-induced EMT (Thakur et al., 2015).

Cetyltrimethylammonium bromide (CTAB) has a non-polar hydrophobic tail and a positively charged polar head (Bag et al., 2016). This structure allows it to attach to cell membranes and form nanopores (Wu et al., 2016). Therefore, CTAB may disrupt lipid bilayers and increase the permeability of cellular membranes, resulting in a change in the intracellular AMP: ATP ratio. $\mathrm{CTAB}$ is a good candidate for an anti-tumor agent (Zhang et al., 2015). CTAB could prevent the metastasis of hepatic cancer through targeting MMP2 and MMP9. CTAB is involved in the suppression of TGF- $\beta$-mediated mesenchymal phenotype and could be a potent medical agent for use in controlling the migration and invasion of hepatic adenocarcinoma ( Wu et al., 2019). What is more, our previous study demonstrated that CTAB has anti-tumor effects on liver cancer and enhances the chemosensitivity of breast cancer cells by targeting various signaling pathways. However, the effect of CTAB on breast cancer metastasis is unclear. Based on the data presented above, we speculate that $\mathrm{CTAB}$ may affect breast cancer metastasis.

In the present study, we aimed to determine whether $\mathrm{CTAB}$ inhibits the metastasis of breast cancer to the lungs. We also investigated the effects of CTAB on molecular changes related to EMT in breast cancer cells.

\section{Materials and Methods}

\section{Materials and reagents}

All cells were donated by the Department of Pharmacology, College of Basic Medical Sciences, Jilin University. CTAB (hexadecyltrimethylammonium bromide, Cat. H6269-250G) was purchased from Sigma Aldrich (St Louis, MO, USA); the anti- $\beta$ actin antibody (Cat. 66009-1-1g) was purchased from Proteintech (Rosemont, IL, USA) and antibodies against Ecadherin (Cat. WL01482), N-cadherin (Cat. WL01047), vimentin (Cat. WL01960), Snail (Cat. WL01863) and Twist1 (Cat. WL00997) were purchased from Wanleibio (Shenyang, China).

\section{Cell culture and MTT assays}

MCF-7 cells were treated with CTAB at final concentrations of $0,2.74 \times 10^{-4}, 5.49 \times 10^{-4}, 8.23 \times 10^{-4}, 13.7 \times 10^{-4}, 20.6 \times$ $10^{-4}, 41.2 \times 10^{-4}, 82.3 \times 10^{-4}$, and $165 \times 10^{-4} \mathrm{mM}$ for $24 \mathrm{~h}$. MDA-MB-231 and 4T1 cells were treated with CTAB at final concentrations of $0,1.37 \times 10^{-4}, 2.47 \times 10^{-4}, 5.21 \times$ $10^{-4}, 10.4 \times 10^{-4}, 20.6 \times 10^{-4}, 41.2 \times 10^{-4}, 82.3 \times 10^{-4}$ and $165 \times 10^{-4} \mathrm{mM}$ for $24 \mathrm{~h}$. MTT assays were performed as previously described (Pan et al., 2019).

\section{Wound-healing assays}

Wound-healing assays were performed as previously described (Pan et al., 2019). MCF-7, MDA-MB-231 and 4T1 cells were treated with $8.23 \times 10^{-4} \mathrm{mM}, 20.6 \times 10^{-4} \mathrm{mM}$, and $5.21 \times 10^{-4} \mathrm{mM}$ CTAB, respectively, for $24 \mathrm{~h}$. The results were analyzed using Image $J$ (National Institutes of Health, Bethesda, MD, USA).

\section{Transwell assays}

Transwell assays were performed as previously described (Shao et al., 2017). MCF-7 cells were treated with $8.23 \times 10^{-4} \mathrm{mM}$
CTAB for 24 h, MDA-MB-231 cells were treated with $20.6 \times$ $10^{-4} \mathrm{mM}$ CTAB for $16 \mathrm{~h}$ and $4 \mathrm{~T} 1$ cells were treated with $5.21 \times 10^{-4} \mathrm{mM}$ CTAB for $16 \mathrm{~h}$. The results were analyzed using Image $\mathrm{J}$.

\section{Western blotting}

MCF-7, MDA-MB-231 and 4T1 cells were treated with $8.23 \times$ $10^{-4} \mathrm{mM}, 20.6 \times 10^{-4} \mathrm{mM}$ and $5.21 \times 10^{-4} \mathrm{mM} \mathrm{CTAB}$, respectively, for $24 \mathrm{~h}$. Total protein was extracted from the cells and from breast tissue of control and CTAB-treated mice with $4 \mathrm{~T} 1$ orthotopic breast cancer. Western blotting was performed as previously described (Tao et al., 2020).

RNA isolation and quantitative reverse transcription PCR MCF-7 cells were treated with $8.23 \times 10^{-4} \mathrm{mM} \mathrm{CTAB} \mathrm{for} 24 \mathrm{~h}$. Total RNA was extracted from the cells and from breast tissue of control and CTAB-treated mice with $4 \mathrm{~T} 1$ orthotopic breast cancer. Quantitative reverse transcription PCR was performed as previously described (Parvani et al., 2015).

\section{Animals}

$\mathrm{BALB} / \mathrm{c}$ mice were purchased from Liaoning Changsheng Biotechnology Co., Ltd. (Benxi, China). All experiments were performed in accordance with the China Public Health Service Guide for the Care and Use of Laboratory Animals. Experiments involving mice and associated protocols were approved by the Dalian University of Technology. 4T1 cells were injected into female $\mathrm{BALB} / \mathrm{c}$ mice. Mice were divided into control and CTAB groups. Mice in the control group were treated with saline and those in the CTAB group were treated with $13.7 \mathrm{mmol} / \mathrm{kg} \mathrm{CTAB}$, both of which were injected into the tail vein every 3 days for 30 days. Mouse body weight was measured every 3 days. After sacrificing the mice, serum, breast tissue and other organs were harvested (Pan et al., 2019).

\section{Statistical analysis}

All experiments were performed on three independent occasions. The experimental data were analyzed using GraphPad Prism 8.0 statistical analysis software (GraphPad, San Diego, CA, USA). Data are expressed as the mean \pm standard deviation (SD) from at least three independent experiments. A two-tailed Student's $t$-test or one-way analysis of variance was used for statistical analyses. $P$-value $<0.05$ was deemed statistically significant.

\section{Results}

$C T A B$ inhibited the metastasis of breast cancer cells in vitro Our previous study showed that CTAB is an anti-cancer agent against various types of cancer. To investigate the effect of $\mathrm{CTAB}$ on breast cancer metastasis, we selected the human breast cancer cells MCF-7 and MDA-MB-231 and the mouse breast cancer cells 4T1. Wound-healing assays showed that the breast cancer cells spread slower to the wound area when they were treated for $24 \mathrm{~h}$ with non-toxic concentrations of CTAB (MCF-7 cells, $8.23 \times 10^{-4} \mathrm{mM}$ CTAB; MDA-MB-231 cells, $20.6 \times 10^{-4} \mathrm{mM} \mathrm{CTAB;} \mathrm{4T1}$ cells, $5.21 \times 10^{-4} \mathrm{mM}$ CTAB; Figs. 1A-1C). Moreover, quantitative data showed that $\mathrm{CTAB}$ significantly inhibited 
(A)

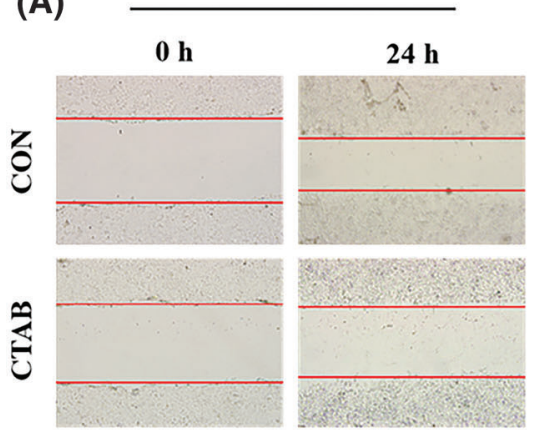

(B)

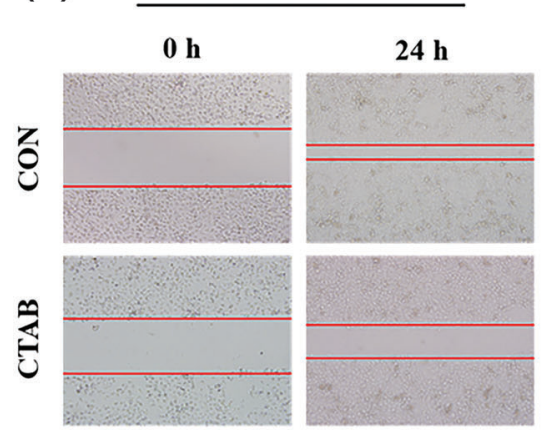

(C)

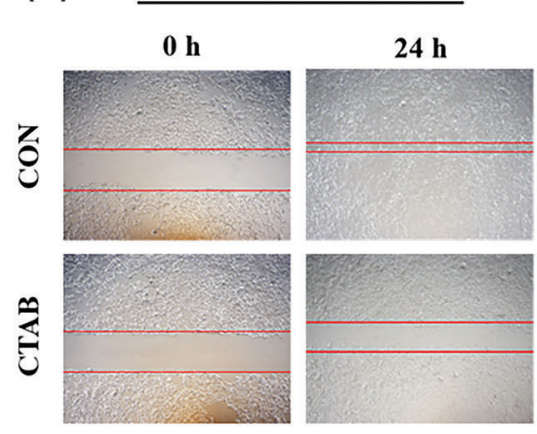

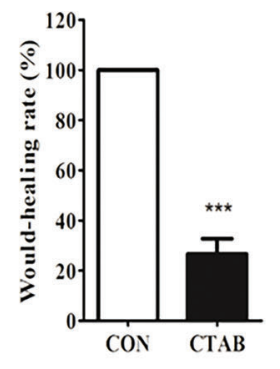
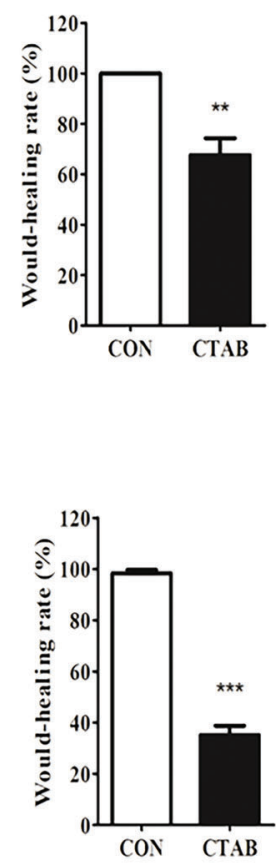

(D)
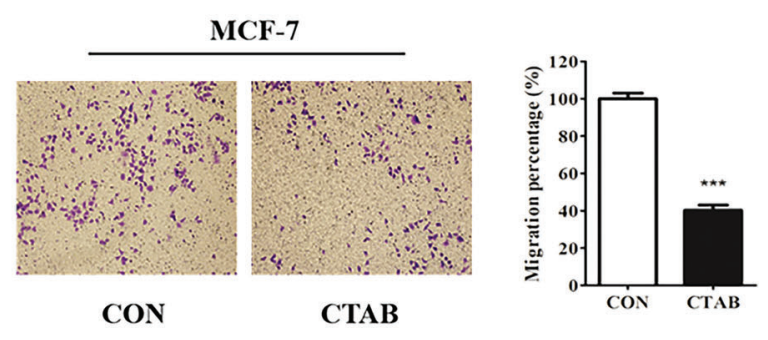

(E)
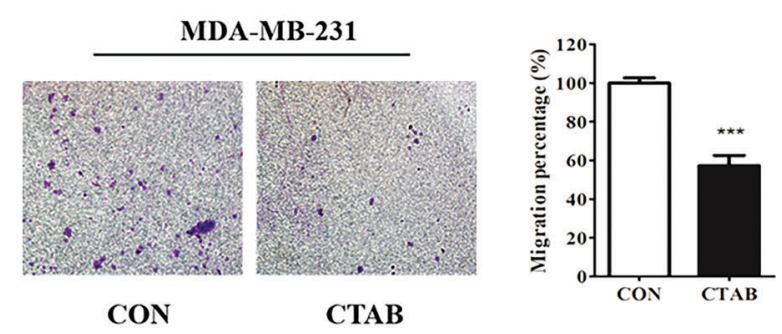

(F)
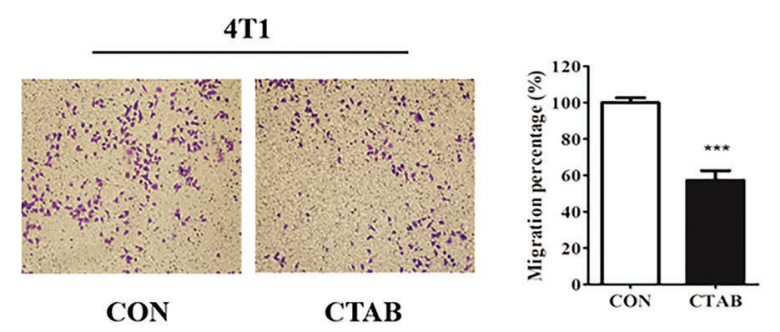

FIGURE 1. CTAB inhibited the metastasis of breast cancer cells in vitro. (A-C) Migration toward the wound area in MCF-7, MDA-MB-231 and $4 \mathrm{~T} 1$ cells treated with CTAB for $24 \mathrm{~h}$. (D, E and F) Migration of MCF-7, MDA-MB-231 and 4T1 cells in transwell assays after treatment with CTAB for 24,16 and 16 h, respectively. Image magnification: $200 \times$. Data are expressed as mean \pm SD. ${ }^{\star} P<0.05,{ }^{* *} P<0.01$, ${ }^{* *} P<0.001 v$ s. controls.

the motility of MCF-7, MDA-MB-231 and 4T1 cells (Figs. $1 \mathrm{~A}-1 \mathrm{C})$. Transwell migration assays showed that $\mathrm{CTAB}$ decreased the number of breast cancer cells passing through the polyester fiber membrane (Figs. 1D-1F). Importantly, the concentration of CTAB used in these experiments did not affect the viability of the breast cancer cells (Fig. S1). These results suggest that $\mathrm{CTAB}$ inhibited the metastasis of breast cancer cells in vitro without causing cell death.

CTAB inactivated the EMT signaling pathway in breast cancer cells

The activation of EMT facilitates cancer metastasis, and Ecadherin, N-cadherin, vimentin, Snail and Twist1 are classical biomarkers of EMT. To investigate the inhibitory effect of CTAB on EMT in breast cancer cells, we measured the protein levels of biomarkers involved in EMT. MCF-7 cells were treated with a non-toxic concentration of CTAB for $24 \mathrm{~h}$. Western blotting results showed that CTAB upregulated the expression levels of E-cadherin and downregulated the expression levels of $\mathrm{N}$-cadherin, vimentin, SNAIL and TWIST1 (Fig. 2A, Fig. S2A). Consistent with the results in MCF-7 cells, CTAB also inhibited the EMT signaling pathway in MDA-MB-231 (Fig. 2B) and 4T1 cells (Fig. 2C). In summary, these findings suggest that CTAB suppressed the activation of EMT signaling in breast cancer cells in vitro.

\section{$C T A B$ inhibited metastasis by activating AMPK in vitro}

Recent studies have indicated that AMPK plays an important role in cancer cell metastasis. Our results showed that CTAB increased the levels of p-AMPK, indicating that it may activate the AMPK signaling pathway (Fig. 3A). To determine the effect of AMPK on breast cancer metastasis, we used the CTAB inhibitor compound $\mathrm{C}$. The addition of compound $\mathrm{C}$ to the wound-healing assays alleviated the inhibition of motility caused by CTAB (Figs. 3C and 3D). Consistent with the wound-healing assay results, treatment with compound $\mathrm{C}$ increased the number of cells passing through the membrane that was decreased by CTAB in transwell migration assays (Figs. $3 \mathrm{E}$ and $3 \mathrm{~F}$ ). CTAB 

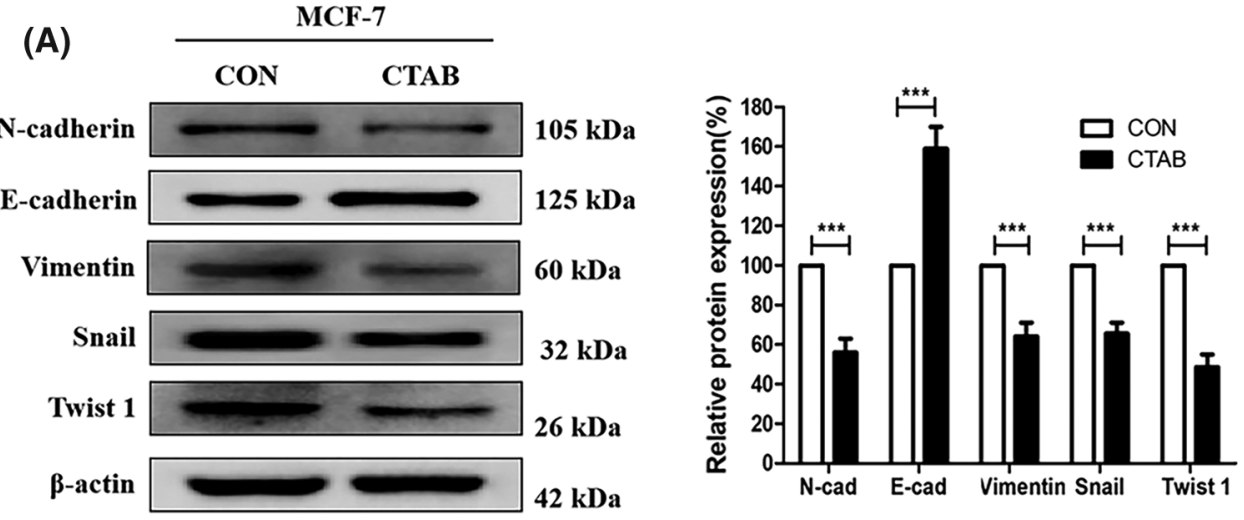

(B)
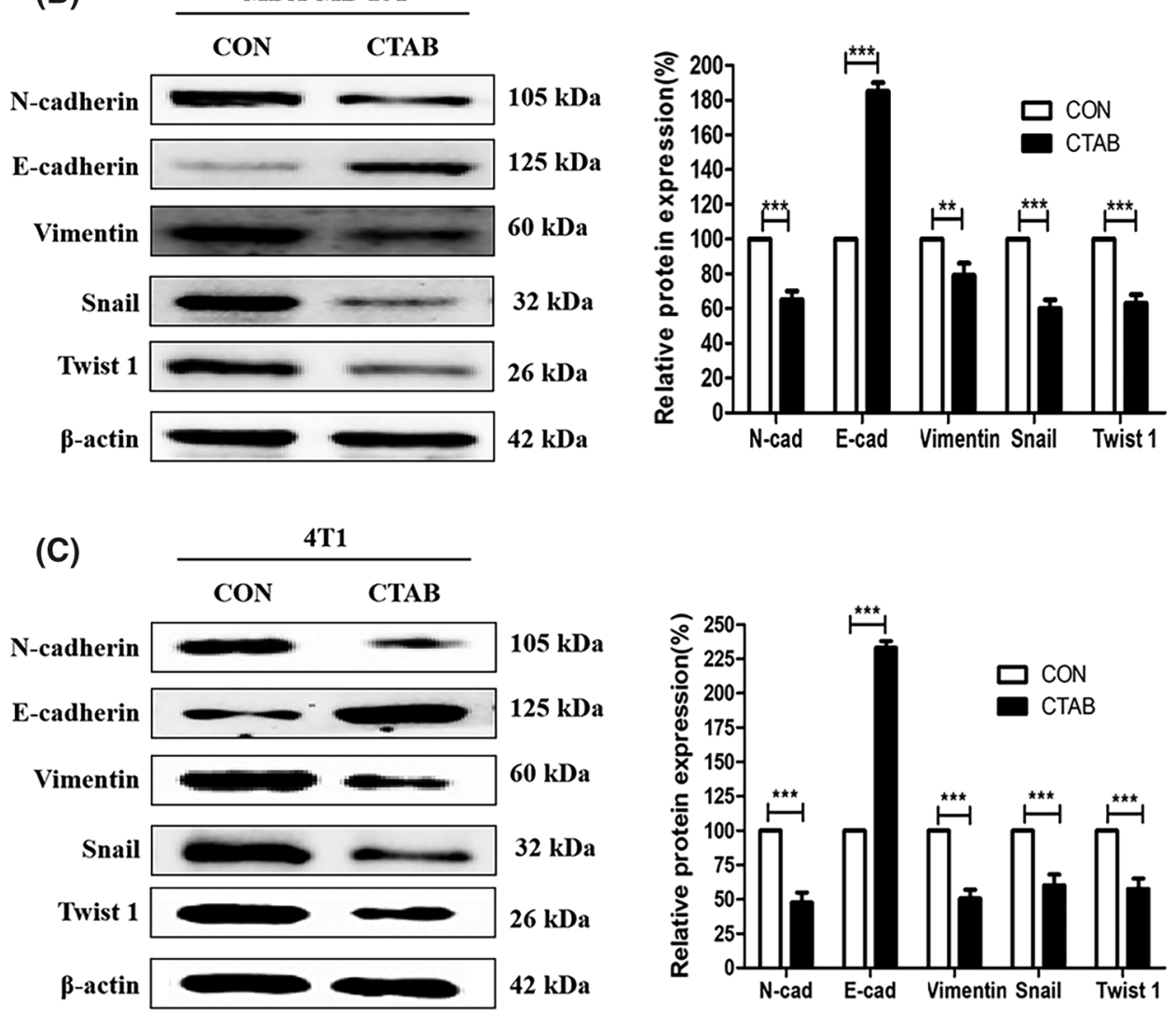

FIGURE 2. CTAB suppressed EMT in breast cancer cells in vitro. (A-C) Protein expression levels of EMTrelated markers after treatment of MCF-7 (A), MDA-MB-231 (B) and (C) $4 \mathrm{~T} 1$ cells with CTAB for $24 \mathrm{~h}$. Image magnification: $200 \times$. Data are expressed as mean \pm SD. ${ }^{\star} P<0.05$, ${ }^{* *} P<0.01,{ }^{* * *} P<0.001$ vs. controls.

inhibited the EMT process related to breast cancer metastasis, but treatment with compound $\mathrm{C}$ reactivated EMT (Figs. 3G and $3 \mathrm{H}$ ). In summary, CTAB inhibited metastasis and the EMT process by activating AMPK in vitro.

$C T A B$ inhibited the metastasis of breast cancer to the lungs in vivo The experiments described above showed that CTAB inhibited the metastasis of breast cancer cells in vitro. To explore the effect of $\mathrm{CTAB}$ on breast cancer metastasis in vivo, we established a $4 \mathrm{~T} 1$ orthotopic breast cancer model in $\mathrm{BALB} / \mathrm{c}$ mice. After 30 days, the primary orthotopic breast tumor metastasized to lung tissue. As shown in Fig. 4A, mice in the control group had many visible metastatic nodes throughout the lungs. However, CTAB treatment reduced the size and number of metastatic nodes in the lungs. In hematoxylin and eosin staining assays, the metastatic lesions were stained dark blue due to nuclear aggregation in cancer cells. The area of staining and the number of cells, stained dark blue, were greater in the control group than in the CTAB-treated group (Fig. 4B). These results indicated that $\mathrm{CTAB}$ inhibited the metastasis of breast cancer to the lungs in vivo. As shown in Fig. $4 \mathrm{C}$ and Fig. $\mathrm{S} 2 \mathrm{~B}, \mathrm{CTAB}$ significantly increased E-cadherin protein levels and decreased N-cadherin, vimentin, Snail and Twist1 protein levels. Consistent with the in vitro data, $\mathrm{CTAB}$ inhibited the EMT signaling pathway in vivo. Furthermore, we measured AMPK activation in vivo and found that the levels of $\mathrm{p}$-AMPK were significantly increased after CTAB treatment (Fig. 4D). These data further confirmed that CTAB suppressed the metastasis of breast cancer to the lungs by targeting AMPK.

\section{The safety of CTAB treatment}

Besides the obvious therapeutic efficacy of CTAB, systemic safety is another important factor to evaluate when determining 

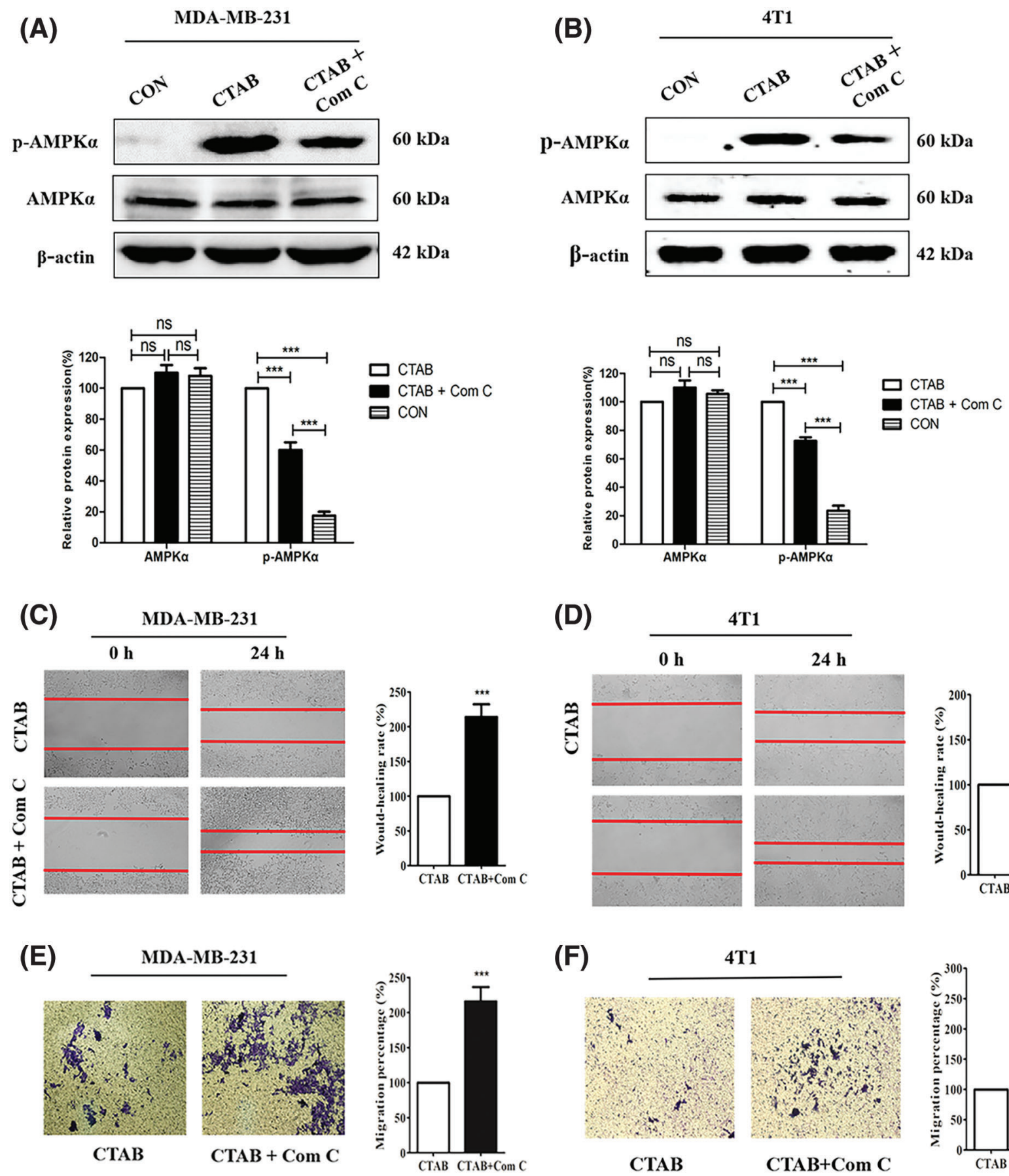

(F)
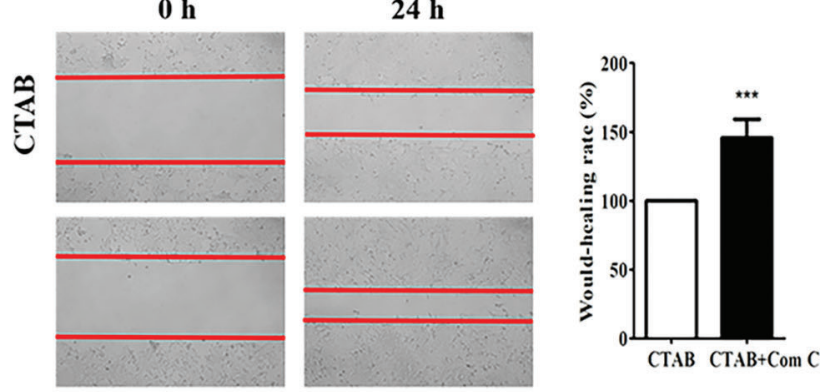

FIGURE 3. (Continued)
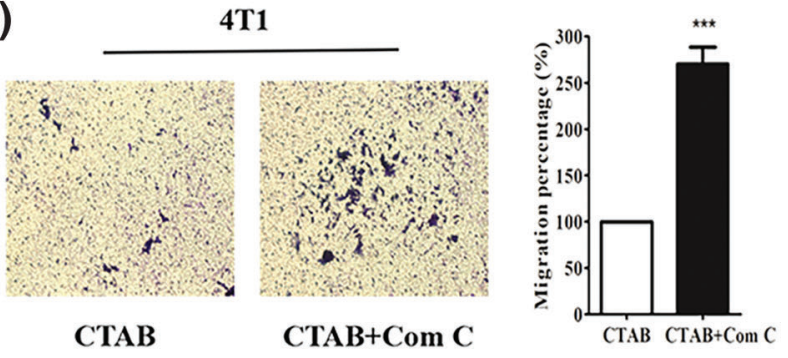

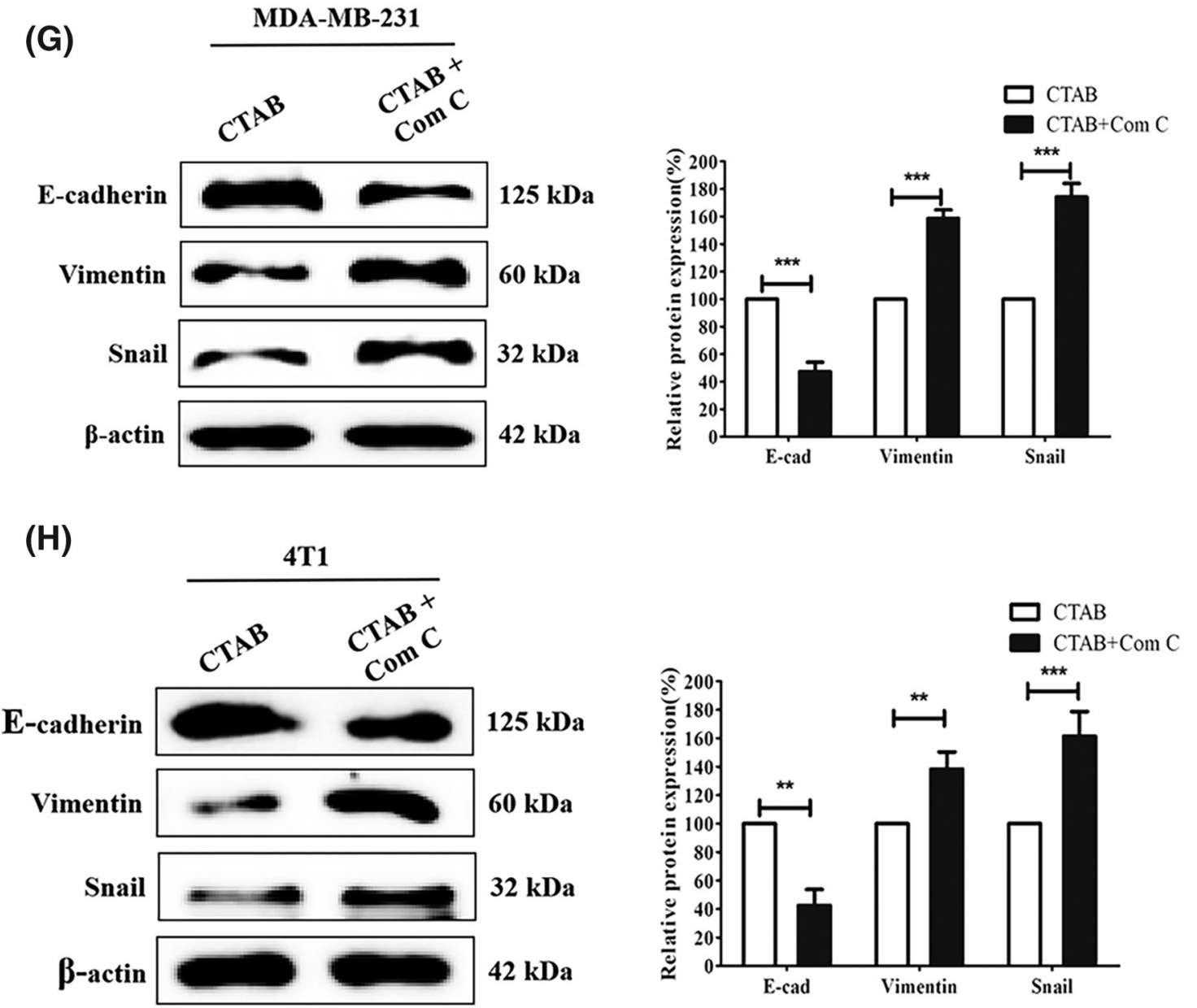

FIGURE 3. CTAB inhibited metastasis by activating AMPK in vitro. (A-B) AMPK and p-AMPK levels after treatment of MDA-MB-231 and $4 \mathrm{~T} 1$ cells with $\mathrm{CTAB}$ or CTAB combined with compound C for $24 \mathrm{~h}$. (C-D) Migration of MDA-MB-231 and $4 \mathrm{~T} 1$ cells toward the wound area after treatment with CTAB or CTAB combined with compound C for $24 \mathrm{~h}$. (E-F) Migration of MDA-MB-231 and 4T1 cells in transwell assays after treatment with CTAB or CTAB combined with compound $\mathrm{C}$ for $24 \mathrm{~h}$. (G-H) Protein expression levels of EMT-related markers in MDAMB-231 and 4T1 cells treated with CTAB or CTAB combined with compound C for $24 \mathrm{~h}$. Image magnification: 200×. Data are expressed as mean $\pm \mathrm{SD} .{ }^{\star} P<0.05,{ }^{* *} P<0.01,{ }^{* *} P<0.001$ vs. controls.

whether CTAB is a good candidate for breast cancer metastasis therapy. Alanine aminotransferase (ALT) and aspartate aminotransferase (AST) are enzymes required for the metabolism of sugars and proteins in the human body. Blood urea nitrogen (BUN), which is filtered out by the glomerulus, is one of the indicators used to evaluate renal function. Therefore, ALT, AST and BUN levels are recognized as indices to evaluate safety. We measured the body weight of mice and performed blood chemistry analyses, including the measurement of AST and ALT levels to assess hepatic function and BUN levels to assess renal function. There were no significant differences in body weight or AST, ALT or BUN levels between control and CTAB-treated mice (Figs. 5A-5D). These results indicated that $\mathrm{CTAB}$ was a safe and effective agent for breast cancer metastasis therapy. Therefore, CTAB has great potential for use in breast cancer treatment.

\section{Discussion}

With the malignant development of cancer cells, the acquisition of invasive abilities plays an important role in cancer metastasis. Many studies have demonstrated that EMT is involved in the process of cancer metastasis
(Shi et al., 2020; Wang et al., 2020). EMT is activated during the metastasis of many types of cancer, such as primary liver, bladder, lung and colon cancer and malignant melanoma. In addition to its role in cancer metastasis, EMT is also associated with cancer recurrence and drug resistance (Parvani et al., 2015; Wheelock et al., 2008). Cancer stem cells (CSCs) are capable of self-renewal and can differentiate into a variety of cancer cell types (Wang et al., 2018). The acquisition of stem-like characteristics serves as a critical driver of cancer metastasis. Furthermore, EMT is highly activated in CSCs and promotes their development (Hong et al., 2018). Yue et al. (2018) showed that LGR4 regulates CSCs by targeting the EMT process. Kusoglu et al. (2020) reported that EMT is activated during breast cancer metastasis, and the inhibition of EMT decreases the stemness of metastatic breast cancer cells. Our results showed that CTAB suppressed EMT and thus inhibited breast cancer metastasis.

Abnormal energy metabolism is related to the malignancy of cancer cells and promotes cancer proliferation, metastasis, and drug resistance (Park et al., 2020). In particular, metabolic reprogramming in CSCs contributes to their rapid growth and self-renewal (Abd-El-Raouf et al., 2020). Recently, 

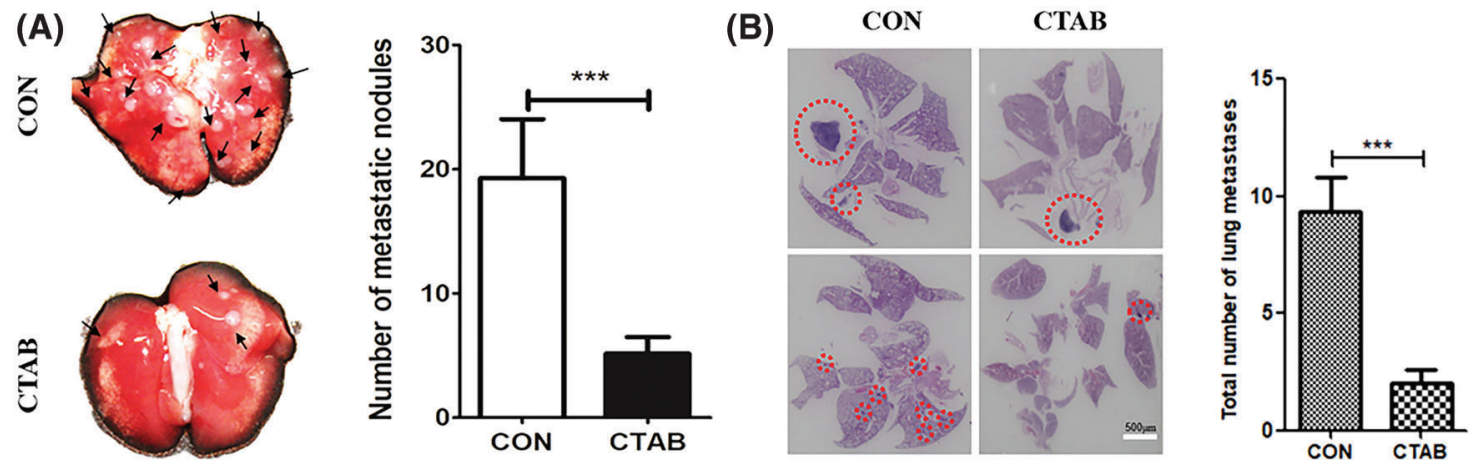

(C)
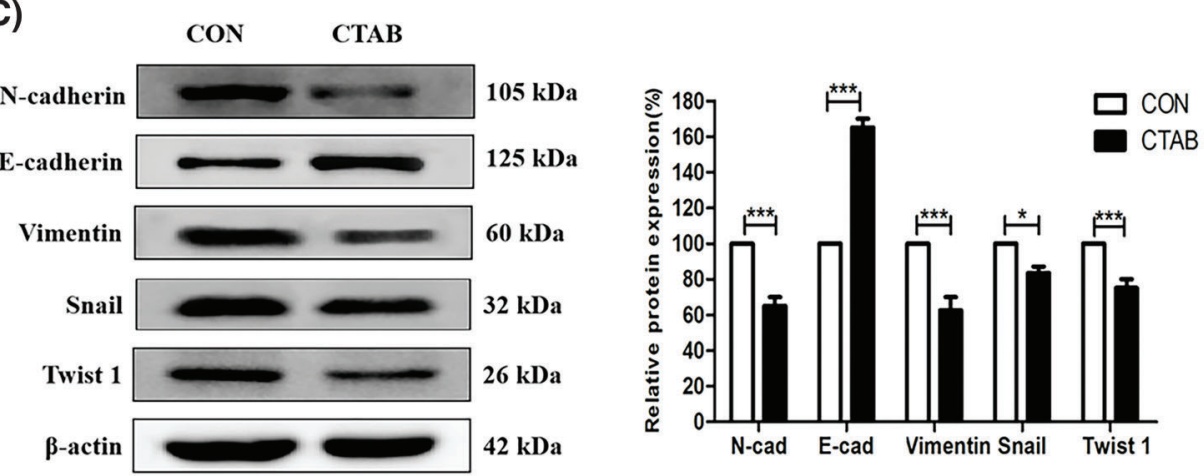

(D)
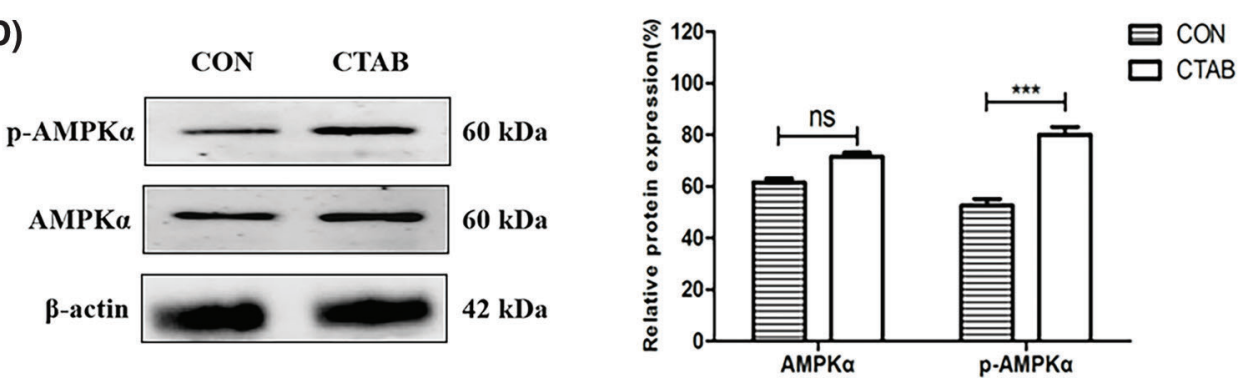

FIGURE 4. CTAB inhibited the metastasis of breast cancer to the lungs in vivo. (A) Typical images of lung tissues from mice treated with CTAB. (B) Histological examination of metastatic lesions in the lung tissue (hematoxylin and eosin staining) of mice treated with CTAB (scale bar = $500 \mu \mathrm{m}$ ). (C) Protein expression levels of EMT-related markers in the mammary tissue of mice treated with CTAB. (D) AMPK and p-AMPK levels in the mammary tissue of mice treated with CTAB. Data are expressed as mean $\pm \mathrm{SD} .{ }^{\star} P<0.05,{ }^{* *} P<0.01$, ${ }^{* *} P<0.001 v s$. controls.

the regulation of energy metabolism in CSCs by targeting HIF, c-Myc, p53 and PI3K has shown anti-tumor effects. EMT is also a target for cancer metabolic reprograming and is regulated by Snail, Dlx-2, HIF-1 $\alpha$, STAT3, TGF- $\beta$, Wnt and Akt (Lee et al., 2018).

AMPK plays a key role in regulating energy metabolism by coordinating glucose and lipid metabolism. AMPK is activated by alterations in the cancer microenvironment, after which it maintains intracellular ATP levels in a steady state (Juszczak et al., 2020). As a sensor of energy metabolism, AMPK is closely related to cancer development. AMPK is a cancer therapy target in various types of cancer due to its roles in proliferation, metastasis, and drug resistance. Alterations in the ADP: ATP ratio activate AMPK. Our previous study showed that CTAB has antitumor effects on hepatocellular carcinoma and can enhance the chemosensitivity of cancer cells (Pan et al., 2019). As an antineoplastic drug, $\mathrm{CTAB}$ attaches to cellular membranes and forms nanopores, which may increase the permeability of the mitochondrial membrane. Due to its effect on membrane permeability, $\mathrm{CTAB}$ may regulate the responses to physiological cues, such as hypoxia and TGF- $\beta$ treatment (Wang et al., 2018). Moreover, the phosphorylation of AMPK upregulates the expression and nuclear localization of Twist1, which is involved in the EMT process. Dong et al. found that the activation of AMPK/mTOR signaling protects papillary thyroid cancer cells from high glucoseinduced EMT and cellular senescence (Dong et al., 2017). AMPK may be a target for cancer metastasis treatment, as it regulates cancer energy metabolism by inhibiting EMT. $\mathrm{CTAB}$, as a compound that affects ATP synthesis, may activate $A M P K$ and thus may be a candidate for cancer therapy. CTAB has previously been reported to compromise bio-energetic homeostasis by inhibiting $\mathrm{H}^{+}$-ATP synthase to prevent mitochondrial repolarization, leading to a progressive reduction in intracellular ATP levels in human head and neck cancer. Previous studies by our group and others have shown that CTAB has anti-tumor effects and can enhance the chemosensitivity of cancer cells (Pan et al., 2019). In addition, we previously showed that CTAB 
(A)

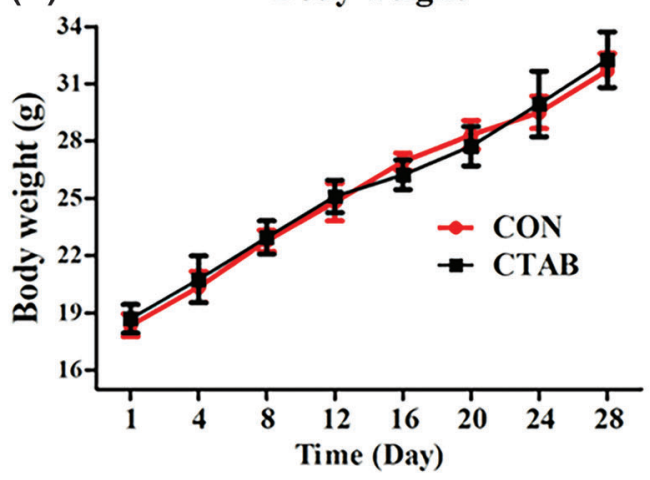

(C)

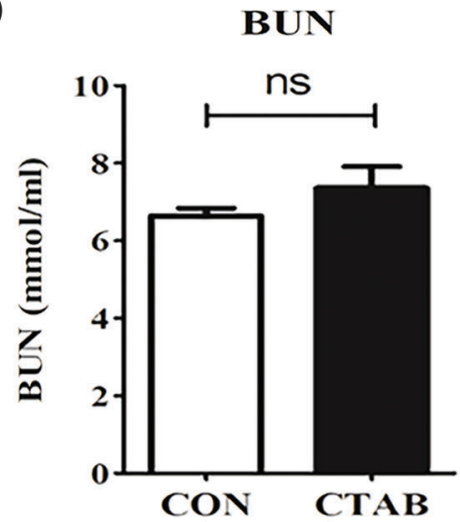

(B)

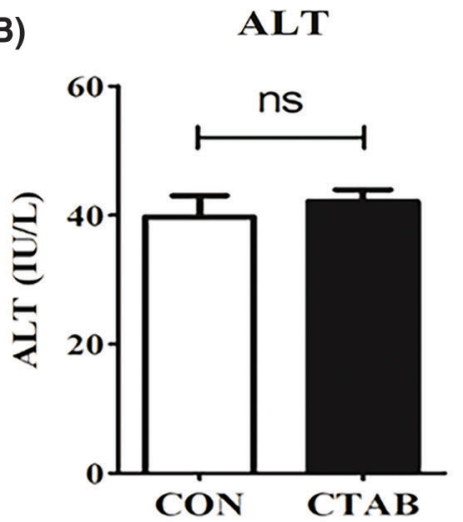

(D)

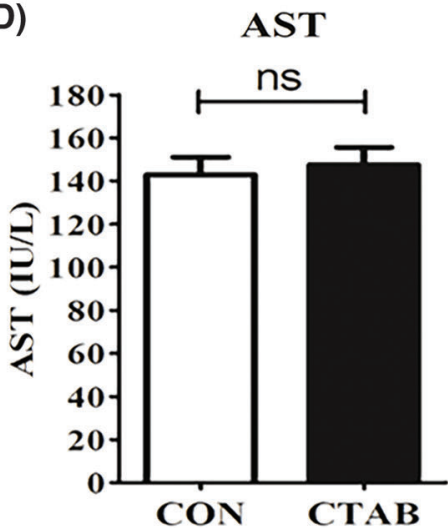

FIGURE 5. The safety of CTAB treatment. (A) Mouse body weight after treatment with CTAB. (B) ALT, (C) AST and (D) BUN levels in mice treated with CTAB. Data are expressed as mean $\pm \mathrm{SD}$. ${ }^{*} P<$ $0.05,{ }^{*} P<0.01,{ }^{* *} P<0.001$ vs. controls. decreases the resistance of breast cancer to doxorubicin chemotherapy by activating the AMPK signaling cascade in vitro and in vivo, and that $\mathrm{CTAB}$ induces mitochondrial apoptosis in human hepatocarcinoma HepG2 cells by activating AMPK and p53 signaling pathways. Thus, CTAB may also inhibit breast cancer metastasis by targeting AMPK.

\section{Conclusions}

In conclusion, we provide evidence that $\mathrm{CTAB}$ suppresses breast cancer metastasis by inhibiting EMT in vitro and in vivo. These data may be important in facilitating the development of improved therapies for breast cancer.

Authors' Contribution: The authors confirm contribution to the paper as follows: study conception and design: Yue Pan, Ning Li; data collection: Yang Chen; analysis and interpretation of results: Yongjie Yang, Shuhan Lyu; draft manuscript preparation: Ning Li, and Yue Pan. All authors reviewed the results and approved the final version of the manuscript.

Availability of Data and Materials: The datasets generated during and/or analysed during the current study are available from the corresponding author on reasonable request.

Ethics Approval: This research was approved by Biological and Medical Ethics Committee of Dalian University of Technology (Approval code: 2021-094; Approval date: 15 January, 2021).

Funding Statement: This research was supported by 81803024 (National Natural Science Foundation of China) and DUT21LK23 (Fundamental Research Funds for the Central Universities).
Conflicts of Interest: The authors declare that they have no conflicts of interest to report regarding the present study.

\section{References}

Abd-El-Raouf R, Ouf SA, Gabr MM, Zakaria MM, El-Yasergy KF, Ali-El-Dein B (2020). Escherichia coli foster bladder cancer cell line progression via epithelial mesenchymal transition, stemness and metabolic reprogramming. Scientific Reports 10: 18024 .

Bag S, Chaudhury S, Pramanik D, Dasgupta S, Dasgupta S (2016). Hydrophobic tail length plays a pivotal role in amyloid beta (25-35) fibril-surfactant interactions. Proteins: Structure, Function, and Bioinformatics 84: 1213-1223.

Comijn J, Berx G, Vermassen P, Verschueren K, Van Grunsven L et al. (2001). The two-handed $\mathrm{E}$ box binding zinc finger protein SIP1 downregulates E-cadherin and induces invasion. Molecular Cell 7: 1267-1278.

Chiu YC, Shieh DC, Tong KM, Chen CP, Huang KC et al. (2009). Involvement of AdipoR receptor in adiponectin-induced motility and $\alpha 2 \beta 1$ integrin upregulation in human chondrosarcoma cells. Carcinogenesis 30: 1651-1659.

Dong D, Cai GY, Ning YC, Wang JC, Lv Y et al. (2017). Alleviation of senescence and epithelial-mesenchymal transition in aging kidney by short-term caloric restriction and caloric restriction mimetics via modulation of $\mathrm{AMPK} / \mathrm{mTOR}$ signaling. Oncotarget 8: 16109.

Fu J, Zhang L, He T, Xiao X, Liu X et al. (2012). TWIST represses estrogen receptor-alpha expression by recruiting the NuRD protein complex in breast cancer cells. International Journal of Biological Sciences 8: 522-532. DOI 10.7150/ijbs.4164.

Gordan JD, Thompson CB, Simon MC (2007). HIF and c-Myc: Sibling rivals for control of cancer cell metabolism and 
proliferation. Cancer Cell 12: 108-113. DOI 10.1016/j. ccr.2007.07.006.

Greenburg G, Hay ED (1982). Epithelia suspended in collagen gels can lose polarity and express characteristics of migrating mesenchymal cells. Journal of Cell Biology 95: 333-339. DOI 10.1083/jcb.95.1.333.

Hong D, Fritz AJ, Zaidi SK, van Wijnen AJ, Nickerson JA et al. (2018). Epithelial-to-mesenchymal transition and cancer stem cells contribute to breast cancer heterogeneity. Journal of Cellular Physiology 233: 9136-9144. DOI 10.1002/jcp.26847.

Juszczak F, Caron N, Mathew AV, Declèves AE (2020). Critical role for AMPK in metabolic disease-induced chronic kidney disease. International Journal of Molecular Sciences 21: 7994. DOI 10.3390/ijms21217994.

Koual M, Tomkiewicz C, Cano-Sancho G, Antignac JP, Bats AS, Coumoul X (2020). Environmental chemicals, breast cancer progression and drug resistance. Environmental Health 19: 117. DOI 10.1186/s12940-020-00670-2.

Kusoglu A, Bagca BG, Ay NPO, Gunduz C, Avci CB (2020). Telomerase inhibition regulates EMT mechanism in breast cancer stem cells. Gene 759: 145001. DOI 10.1016/j.gene.2020.145001.

Lasagna M, Hielpos MS, Ventura C, Mardirosian MN, Martin G et al. (2020). Chlorpyrifos subthreshold exposure induces epithelial-mesenchymal transition in breast cancer cells. Ecotoxicology and Environmental Safety 205: 111312. DOI 10.1016/j.ecoenv.2020.111312.

Lee SY, Ju MK, Jeon HM, Lee YJ, Kim CH et al. (2018). Oncogenic metabolism acts as a prerequisite step for induction of cancer metastasis and cancer stem cell phenotype. Oxidative Medicine and Cellular Longevity 2018: 1-28. DOI 10.1155/2018/1027453.

Liang Y, Zhang H, Song X, Yang Q (2020). Metastatic heterogeneity of breast cancer: Molecular mechanism and potential therapeutic targets. Seminars in Cancer Biology 60: 14-27. DOI 10.1016/j.semcancer.2019.08.012.

Pan Y, Zhang Y, Chen Q, Tao X, Liu J, Xiao GG (2019). CTAB enhances chemo-sensitivity through activation of AMPK signaling cascades in breast cancer. Frontiers in Pharmacology 10: 843. DOI 10.3389/fphar.2019.00843.

Park JH, Pyun WY, Park HW (2020). Cancer metabolism: Phenotype, signaling and therapeutic targets. Cells 9: 2308. DOI 10.3390/ cells9102308.

Parvani JG, Gujrati MD, Mack MA, Schiemann WP, Lu ZR (2015). Silencing $\beta 3$ integrin by targeted ECO/siRNA nanoparticles inhibits EMT and metastasis of triple-negative breast cancer. Cancer Research 75: 2316-2325. DOI 10.1158/00085472.CAN-14-3485.

Polyak K, Weinberg RA (2009). Transitions between epithelial and mesenchymal states: Acquisition of malignant and stem cell traits. Nature Reviews Cancer 9: 265-273. DOI 10.1038/nrc2620.

Rios Garcia M, Steinbauer B, Srivastava K, Singhal M, Mattijssen F et al. (2017). Acetyl-CoA carboxylase 1-dependent protein acetylation controls breast cancer metastasis and recurrence. Cell Metabolism 26: 842-855.e5. DOI 10.1016/j.cmet.2017.09.018.

Shah N, Mohammad AS, Saralkar P, Sprowls SA, Vickers SD et al. (2018). Investigational chemotherapy and novel pharmacokinetic mechanisms for the treatment of breast cancer brain metastases. Pharmacological Research 132: 47-68. DOI 10.1016/ j.phrs.2018.03.021.

Shao D, Lu MM, Zhao YW, Zhang F, Tan YF et al. (2017). The shape effect of magnetic mesoporous silica nanoparticles on endocytosis, biocompatibility and biodistribution. Acta Biomaterialia 49: 531-540. DOI 10.1016/j.actbio.2016.11.007.

Shi X, Kaller M, Rokavec M, Kirchner T, Horst D, Hermeking H (2020). Characterization of a p53/miR-34a/CSF1R/STAT3 feedback loop in colorectal cancer. Cellular and Molecular Gastroenterology and Hepatology 10: 391-418. DOI 10.1016/j.jcmgh.2020.04.002.

Steinberg GR, Kemp B (2009). AMPK in health and disease. Physiological Reviews 10: 1025-1078. DOI 10.1152/physrev.00011.2008.

Tania M, Khan MA, Fu J (2014). Epithelial to mesenchymal transition inducing transcription factors and metastatic cancer. Tumor Biology 35: 7335-7342. DOI 10.1007/s13277-014-2163-y.

Tao X, Chen Q, Li N, Xiang H, Pan Y et al. (2020). Serotonin-RhoA/ ROCK axis promotes acinar-to-ductal metaplasia in caeruleininduced chronic pancreatitis. Biomedicine \& Pharmacotherapy 125: 109999. DOI 10.1016/j.biopha.2020.109999.

Thakur S, Viswanadhapalli S, Kopp JB, Shi Q, Barnes JL et al. (2015). Activation of AMP-activated protein kinase prevents TGF$\beta 1$-induced epithelial-mesenchymal transition and myofibroblast activation. American Journal of Pathology 185: 2168-2180. DOI 10.1016/j.ajpath.2015.04.014.

Wang T, Fahrmann JF, Lee H, Li YJ, Tripathi SC et al. (2018). JAK/STAT3-regulated fatty acid $\beta$-oxidation is critical for breast cancer stem cell self-renewal and chemoresistance. Cell Metabolism 27: 136-150.e135. DOI 10.1016/j.cmet.2017.11.001.

Wang X, Ji S, Ma Y, Xing X, Zhou Y et al. (2020). Vimentin plays an important role in the promotion of breast cancer cell migration and invasion by leucine aminopeptidase 3. Cytotechnology 72: 639-647. DOI 10.1007/s10616-020-00402-x.

Wheelock MJ, Shintani Y, Maeda M, Fukumoto Y, Johnson KR (2008). Cadherin switching. Journal of Cell Science 121: 727-735. DOI 10.1242/jcs.000455.

Wu TK, Chen CH, Pan YR, Hu CW, Huang FM et al. (2019). Cetrimonium bromide inhibits cell migration and invasion of human hepatic SK-HEP-1 cells through modulating the canonical and non-canonical TGF- $\beta$ signaling pathways. Anticancer Research 39: 3621-3631. DOI 10.21873/ anticanres.13510.

Wu Y, Xu M, Chen X, Yang S, Wu H et al. (2016). CTAB-assisted synthesis of novel ultrathin MoSe 2 nanosheets perpendicular to graphene for the adsorption and photodegradation of organic dyes under visible light. Nanoscale 8: 440-450. DOI 10.1039/C5NR05748E.

Yang JX, Li M, Chen XO, Lian QQ, Wang Q et al. (2019). Lipoxin A 4 ameliorates lipopolysaccharide-induced lung injury through stimulating epithelial proliferation, reducing epithelial cell apoptosis and inhibits epithelial-mesenchymal transition. Respiratory Research 20: 192. DOI 10.1186/s12931-019-1158-Z.

Yue Z, Yuan Z, Zeng L, Wang Y, Lai L et al. (2018). LGR4 modulates breast cancer initiation, metastasis, and cancer stem cells. FASEB Journal 32: 2422-2437. DOI 10.1096/fj.201700897R.

Zhang N, Tao J, Hua H, Sun P, Zhao Y (2015). Low-density lipoprotein peptide-combined DNA nanocomplex as an efficient anticancer drug delivery vehicle. European Journal of Pharmaceutics and Biopharmaceutics 94: 20-29. DOI 10.1016/j.ejpb.2015.04.022.

Zheng T, Lu M, Wang T, Zhang C, Du X (2018). NRBE3 promotes metastasis of breast cancer by down-regulating E-cadherin expression. Biochimica et Biophysica Acta (BBA)-Molecular Cell Research 1865: 1869-1877. DOI 10.1016/j.bbamcr.2018.09.003. 
(A)

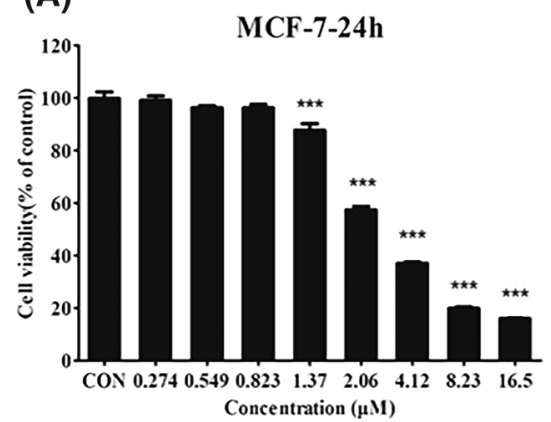

(B)

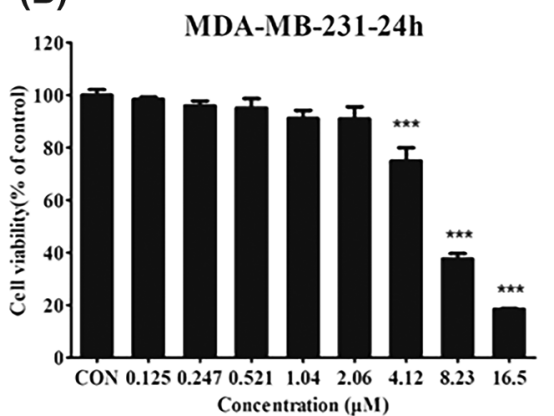

(C)

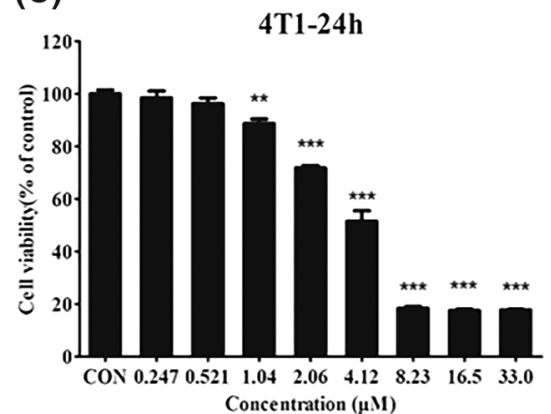

FIGURE S1. (A-C) The viability of MCF-7 (A), MDA-MB-231 (B), and 4T1 (C) cells treated with different concentrations of CTAB. Data are expressed as mean $\pm \mathrm{SD}$. ${ }^{\star} P<0.05,{ }^{* *} P<0.01,{ }^{* *} P<0.001 v$ s. control.

(A)

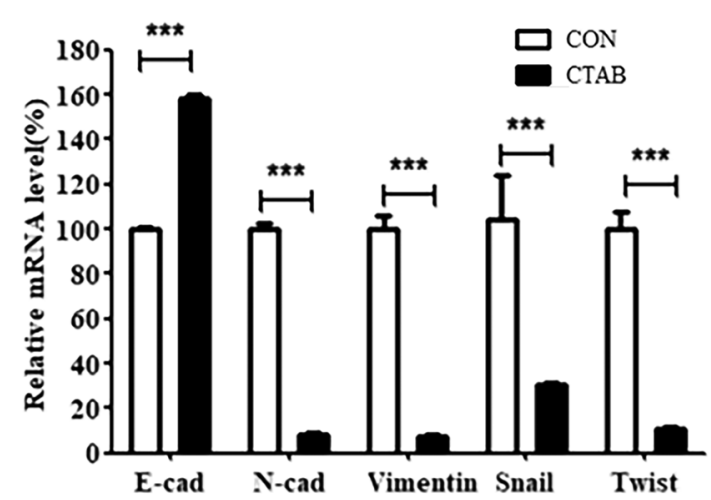

(B)

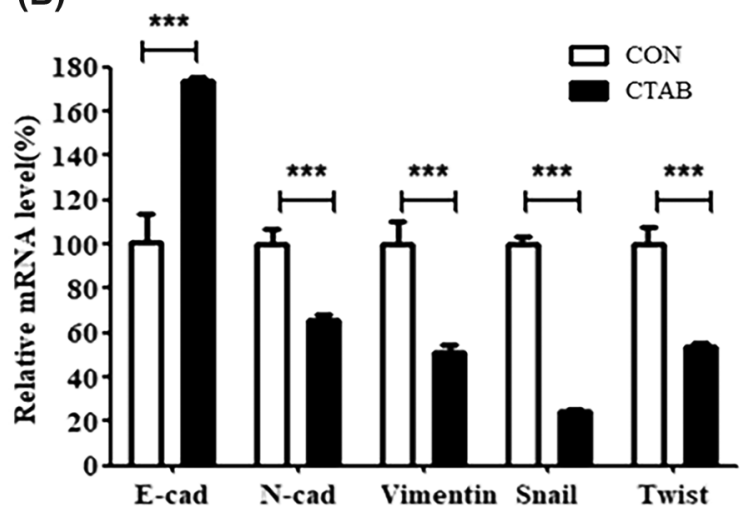

FIGURE S2. (A) mRNA levels of EMT-related genes inMCF-7 cells treated with CTAB for $24 \mathrm{~h}$. (B) mRNA levels of EMT-related genes in vivo after treatment with CTAB. Data are expressed as mean \pm SD. ${ }^{*} P<0.05,{ }^{* *} P<0.01,{ }^{* *} P<0.001 v$ s. controls. 